\title{
Inverse cascade of magnetic helicity in magnetohydrodynamic turbulence
}

\author{
Wolf-Christian Müller, ${ }^{*}$ Shiva Kumar Malapaka, ${ }^{\dagger}$ and Angela Busse ${ }^{\ddagger}$ \\ Max-Planck-Institut für Plasmaphysik, 85748 Garching, Germany
}

(Received 3 May 2011; revised manuscript received 1 December 2011; published 17 January 2012)

\begin{abstract}
The nonlinear dynamics of magnetic helicity $H^{M}$, which is responsible for large-scale magnetic structure formation in electrically conducting turbulent media, is investigated in forced and decaying three-dimensional magnetohydrodynamic turbulence. This is done with the help of high-resolution direct numerical simulations and statistical closure theory. The numerically observed spectral scaling of $H^{M}$ is at variance with earlier work using a statistical closure model [Pouquet et al., J. Fluid Mech. 77, 321 (1976)]. By revisiting this theory, a universal dynamical balance relation is found that includes the effects of kinetic helicity as well as kinetic and magnetic energies on the inverse cascade of $H^{M}$ and explains the above-mentioned discrepancy. Consideration of the result in the context of mean-field dynamo theory suggests a nonlinear modification of the $\alpha$-dynamo effect, which is important in the context of magnetic-field excitation in turbulent plasmas.
\end{abstract}

DOI: 10.1103/PhysRevE.85.015302

PACS number(s): 47.27.Gs, 47.65.Md, 52.65.Kj

The emergence of large-scale magnetic structures in turbulent plasmas is dynamically important in many astrophysical settings such as with regard to the interstellar medium or the magnetic-field generation in planets and stars by the turbulent dynamo effect [1-3]. The structure formation can be studied via the magnetic helicity $H^{M}=\frac{1}{2 V} \int_{V} d V \mathbf{a} \cdot \mathbf{b}$, where $\mathbf{b}$ is the magnetic field and a denotes the magnetic vector potential. This topological characteristic of magnetic fields yields a measure of the linkage and the twist of the field lines [4,5]. In the magnetohydrodynamic (MHD) single-fluid approximation [6], which neglects microscopic scales and the associated kinetic dynamics $H^{M}$, is ideally conserved in a three-dimensional volume with periodic or closed boundary conditions [7]. It is thus prone to a nonlinear and conservative inverse spectral cascade process in the inertial range of MHD plasma turbulence. If driven at small scales $\ell$, the cascade results in spectral transfer of magnetic helicity toward small spatial wave numbers $k \sim \ell^{-1}$ [8], i.e., to the formation of large-scale magnetic structures. This process is thus of fundamental importance with regard to, e.g., the dynamics of magnetic fields in the above-mentioned turbulent astrophysical settings. In spite of its importance, little is known about the nonlinear dynamics that underlies the inverse cascade. It is the purpose of this Rapid Communication to shed some light on the rather mysterious nonlinear phenomenon creating largescale order out of quasirandom turbulent magnetic fluctuations. Please note that although the constraining effect of magnetic helicity conservation on certain $\alpha$-dynamo configurations is important, it is beyond the scope of this work (for further details see Ref. [9]). The existence of such an inverse cascade was first demonstrated in numerical simulations based on the eddy damped quasinormal Markovian (EDQNM) closure model of three-dimensional MHD turbulence [10], which

\footnotetext{
*wolf.mueller@ipp.mpg.de

${ }^{\dagger}$ Present address: Faculty of Mathematics and Physical Sciences, University of Leeds, Leeds LS2 9JT, United Kingdom; shiva.kumar.malapaka@ipp.mpg.de

${ }^{\ddagger}$ Present address: Faculty of Engineering and the Environment, University of Southampton, Southampton SO17 1BJ, United Kingdom; a.busse@soton.ac.uk
}

deals theoretically with the spectral self-similarity of magnetic helicity. The associated self-similar spectral signature in the turbulent inertial range $\sim k^{-2}$ is in agreement with dimensional analysis based on a constant nonlinear spectral flux. Several studies applying direct numerical simulations find an inverse transfer of magnetic helicity (see, e.g., Refs. [11-13]) without reporting self-similar scaling behavior, a notable exception being Ref. [13].

In this Rapid Communication the inverse cascade of magnetic helicity in homogeneous MHD turbulence is studied by three-dimensional high-resolution direct numerical simulations. In the main setup kinetic and magnetic energy and magnetic helicity are injected at small scales of the initially excited spectral range of turbulent fluctuations. It is shown that macroscopic quantities, in particular kinetic helicity and the ratio of kinetic and magnetic energies, have an important influence on the $H^{M}$ cascade that is captured by a universal relation based on dimensional analysis of the MHD EDQNM closure model. This insight suggests a link between magnetic helicity and mean-field dynamo theory in particular with regard to the saturation behavior of the dynamo mechanism.

The dimensionless MHD equations are written as

$$
\begin{gathered}
\partial_{t} \boldsymbol{\omega}=\nabla \times(\mathbf{v} \times \boldsymbol{\omega}+\mathbf{j} \times \mathbf{b}) \\
+\left[\mu_{n}(-1)^{n-1} \Delta^{n}+\lambda \Delta^{-2}\right] \boldsymbol{\omega}+\mathbf{F}_{\mathbf{v}}, \\
\partial_{t} \mathbf{b}=\nabla \times(\mathbf{v} \times \mathbf{b})+\left[\eta_{n}(-1)^{n-1} \Delta^{n}+\lambda \Delta^{-2}\right] \mathbf{b}+\mathbf{F}_{\mathbf{b}}, \\
\nabla \cdot \mathbf{v}=\nabla \cdot \mathbf{b}=\mathbf{0},
\end{gathered}
$$

where $\mathbf{v}$ is the velocity, $\boldsymbol{\omega}=\boldsymbol{\nabla} \times \mathbf{v}$ is the vorticity, $\mathbf{b}$ is the magnetic field, and $\mathbf{j}=\boldsymbol{\nabla} \times \mathbf{b}$ is the electric current density. Equations (1)-(3) are solved by a standard pseudospectral method using a leapfrog scheme for time integration. Antialiasing is achieved by spherical mode truncation. The simulation domain is a triply $2 \pi$-periodic cube discretized by $1024^{3}$ collocation points. Hyperviscous small-scale dissipation operators of order $n=8$ are used to improve scale separation parametrized by the hyperdiffusion coefficients $\mu_{n}$ and $\eta_{n}$ with the hyperviscosity $\mu_{8}=9 \times 10^{-41}$ and $\mu_{8} / \eta_{8}=1$. Boundary effects at smallest spatial wave numbers $k$ are alleviated by a large-scale energy sink $\lambda \Delta^{-2}$ for both fields with the constant $\lambda$ set equal to 0.5 . The forcing terms $\mathbf{F}_{\mathbf{v}}$ and $\mathbf{F}_{\mathbf{b}}$ are random, 


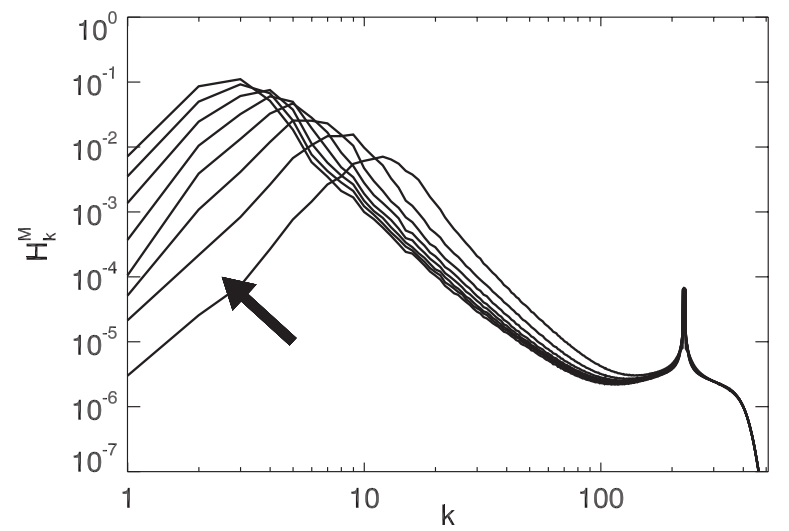

FIG. 1. Inverse cascade of magnetic helicity $H_{k}^{M}$ in threedimensional MHD turbulence for $t \in[1,6.66]$. Curves are given for points in time spread equidistantly over the interval. Magnetic fluctuations with maximal magnetic helicity and nonhelical velocity fluctuations are supplied through the forcing with $k_{0}=206$.

$\delta$-correlated processes of equal amplitude that act over a band of wave numbers $k \in\left[k_{0}-3, k_{0}+3\right]$ with $k_{0}=206$. They inject velocity- and magnetic-field fluctuations with welldefined kinetic and magnetic helicities, kinetic helicity being defined as $H^{K}=\frac{1}{2 V} \int_{V} d V \mathbf{v} \cdot \omega$. Such driving, chosen here for simplicity and numerical efficiency, could, in principle, be realized by a random small-scale distribution of electric currents and forces. The initial velocity and magnetic fields are smooth with equal energies, random phases, and fluctuations that have a Gaussian energy distribution, peaked at $k_{0}$. In the course of the simulation the total energy quickly attains a quasistationary state, fluctuating around unity with $E^{M} / E^{K} \approx$ 9. Cross helicity $H^{C}=\frac{1}{2 V} \int_{V} d V \mathbf{v} \cdot \mathbf{b}$ is negligible. The simulation is carried up to $t=6.66$ large-eddy turnover times. While the application of hyperviscous dissipation operators is necessary to observe well-developed scaling ranges, it precludes the unambiguous definition of a Reynolds number.

The temporal evolution of the magnetic helicity spectrum over the simulation period shown in Fig. 1 indicates inverse spectral transfer. This is also reflected by the nonlinear spectral flux of magnetic helicity

$$
\Pi_{k}^{H^{M}}=2 \int_{0}^{k} d k^{\prime} k^{\prime 2} \int d \Omega\left(\mathbf{b}_{\mathbf{k}^{\prime}}^{*} \cdot[\mathbf{v} \times \mathbf{b}]_{\mathbf{k}^{\prime}}+\text { c.c. }\right),
$$

with $[\cdots]_{\mathbf{k}}$ denoting Fourier transformation and the asterisk standing for complex conjugate (c.c.). The flux spectrum shown in Fig. 2(a) is constant over finite wave-number intervals on both sides of the forcing band signaling equilibrium between turbulence driving and dissipation. While on the right-hand side direct spectral transfer is observed as a result of the small-scale energy sink, on the left-hand side of the forcing band an inverse cascade develops that is driven by the constant magnetic helicity input around $k_{0}$. The spectral flux of total energy $E=E^{K}+E^{M}=\int d^{3} k\left(\left|v_{\mathbf{k}}\right|^{2}+\left|b_{\mathbf{k}}\right|^{2}\right) / 2$ is given by

$$
\begin{aligned}
\Pi_{k}= & \int_{0}^{k} d k^{\prime} k^{\prime 2} \int d \Omega\left(i \mathbf{k}^{\prime} \times[\mathbf{v} \times \boldsymbol{\omega}+\mathbf{j} \times \mathbf{b}]_{\mathbf{k}^{\prime}} \cdot \mathbf{v}_{\mathbf{k}^{\prime}}^{*}\right. \\
& \left.+i \mathbf{k}^{\prime} \times[\mathbf{v} \times \mathbf{b}]_{\mathbf{k}^{\prime}} \cdot \mathbf{b}_{\mathbf{k}^{\prime}}^{*}+\text { c.c. }\right)
\end{aligned}
$$

and is shown in Fig. 2(b). It lacks spectral constancy in the inverse-cascade region and it is principally carried by magnetic

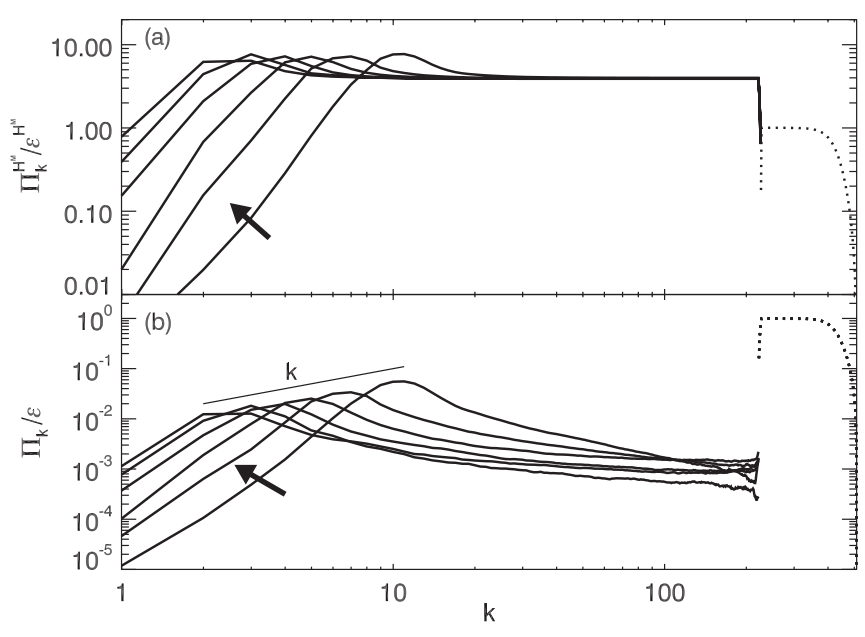

FIG. 2. Moduli of fluxes of (a) magnetic helicity and (b) total energy (inverse, solid curves; direct, dotted curves) normalized by corresponding dissipation rates. Flux spectra are shown for $t \in[1.66,6.66]$ for the forced simulation and are spread equidistantly over this period.

energy transfer. This suggests that the inverse energy flux is a consequence of the inverse cascade of $H^{M}$. The linear scaling of the $\Pi_{k}$ envelope that follows from the dimensional estimate $E_{k} \sim k H_{k}^{M}$ in combination with the approximate constant value of the envelope of $\Pi_{k}^{H^{M}}$ also supports this interpretation.

The compensated magnetic helicity spectrum at the end of the simulation period is displayed in Fig. 3(a). It exhibits two approximate scaling ranges: on the direct transfer side for $250 \lesssim k \lesssim 400$ and in the inverse transfer region for $7 \lesssim k \lesssim$ 30. The corresponding asymptotic scaling laws are $\widetilde{H}_{k}^{M} \approx$ $k^{-3.3}$ (inverse, cf. [13]) and $H_{k}^{M} \sim k^{-1.5}$ (direct). The latter value has to be taken with care due to the very short spectral range and the high-order hyperviscosity acting at largest wave numbers. The inverse-cascade scaling is at variance with the $k^{-2}$ behavior reported in Ref. [10].

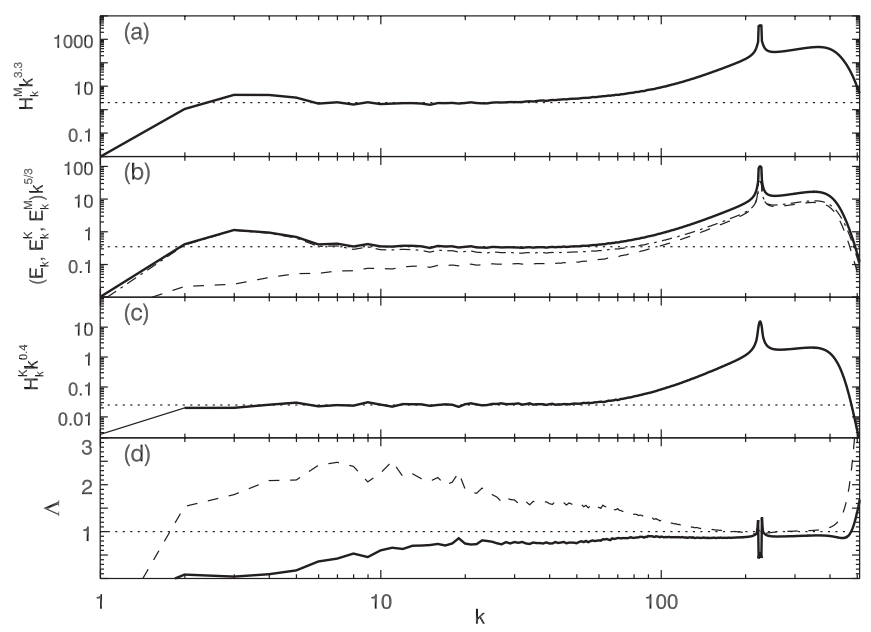

FIG. 3. Compensated spectra of (a) magnetic helicity; (b) total (solid line), kinetic (dashed line), and magnetic (dash-dotted line) energy, and (c) kinetic helicity. (d) $\Lambda=\left(E_{k}^{K} / E_{k}^{M}\right)^{\gamma} H_{k}^{J} / H_{k}^{K}$ for $\gamma=1$ (dashed line) and $\gamma=2$ (solid line) at $t=6.66$ in the driven simulation. 
Since $H^{M}$ is the helicity of the magnetic vector potential a, its inverse cascade pulls quantities derived from this potential, e.g. magnetic energy and to a lesser extent also electric current density $\mathbf{j}_{k} \sim k^{2} \mathbf{a}_{k}$, toward large scales. The magnetic and velocity fields are intrinsically coupled in MHD turbulence by Alfvénic fluctuations [14] and thus similar behavior is observed for the spectral kinetic energy and kinetic helicity $H_{k}^{K}$ as well. The respective spectra are thus expected to inherit the self-similarity from $H_{k}^{M}$ as is indeed observed [see, e.g., Fig. 3(c)]. It seems to be reasonable to regard the merging of current-carrying structures where the currents have significant positively aligned components and thus experience mutual attraction as the physical mechanism of the inverse cascade of magnetic helicity [15]. This is also in line with recent statements in the literature about nonlocality of the magnetic helicity flux $[12,16]$. Terming the inverse transfer of $H_{k}^{M}$ a cascade is thus merely following convention and not a physical description of the actual process of a spectrally non-local merging of current-carrying strucures. Note that in this simulation no kinetic helicity is injected by the turbulence driving. In the case of simultaneous injection of kinetic helicity, the spectral diagnostics discussed in this work show no significant difference from the presented simulations. In general, the details of the small-scale forcing, such as randomness of amplitudes and/or complex phases, were verified to have no measurable impact on the self-similar behavior reported below. The only significant parameter in this respect is the separation of the smallest admissible wave number $k=1$ and the forcing wave number of about two decades. The scale separation determines the extent of the observable self-similar inverse-cascade dynamics. It is thus even more important than the classical Reynolds number, which is not well defined due to the necessary high-order hyperviscous small-scale diffusion.

The energy spectra in Fig. 3(b) exhibit approximate scaling known from decaying turbulence [14,17], i.e., $E_{k} \sim k^{-5 / 3}$ and $E_{k}^{R}=\left|E_{k}^{M}-E_{k}^{K}\right| \sim k^{-7 / 3}$ (not shown), with an excess of magnetic energy. The observations are in agreement with the interpretation of the finite levels of $E_{k}^{K}$ and $H_{k}^{K}$ as a result of the local and temporary stirring induced by changes of magnetic-field topology. This is to be expected in the course of the inverse cascade of magnetic helicity. As will be shown in the following, the lacking equipartition of $E_{k}^{K}$ and $E_{k}^{M}$ and the presence of kinetic helicity are the reasons for disagreement with the above-mentioned EDQNM simulations of Ref. [10]. There, the relaxation time of the nonlinear interaction $\theta_{k p q}$, which represents a free parameter of the EDQNM approach and determines the nonlinear process governing turbulent dynamics, is chosen to be the Alfvén time $\left(k B_{0}\right)^{-1}$. Consequently, the resulting dominance of Alfvénic interactions drives the system in the inertial range into a nearly perfect equipartition of kinetic and magnetic energies.

The observed spectral scaling of magnetic helicity can be better understood with the help of the integro-differential EDQNM equation for the evolution of $H_{k}^{M}$. A formally similar approach has been successful with regard to the residual energy spectrum $E_{k}^{R}=\left|E_{k}^{M}-E_{k}^{K}\right|$ [14]. The equation for the evolution of $H_{k}^{M}$ in the EDQNM model [10] reads

$\left(\partial_{t}+\eta_{1} k^{2}\right) H_{k}^{M}=\int_{\Delta} d p d q \theta_{k p q}\left(T_{k}^{\mathrm{adv}}+T_{k}^{k h l}+T_{k}^{\mathrm{Lor}}\right)$, with

$$
\begin{aligned}
T_{k}^{\mathrm{adv}} & =h_{k p q} \frac{k}{p q}\left(k^{2} E_{q}^{K} H_{p}^{M}-p^{2} E_{q}^{K} H_{k}^{M}\right), \\
T_{k}^{k h l} & =h_{k p q} \frac{k}{p q}\left(\frac{k^{2}}{p^{2}} E_{q}^{M} H_{p}^{K}-\frac{k^{2}}{p^{2}} E_{k}^{M} H_{q}^{K}\right), \\
T_{k}^{\text {Lor }} & =e_{k p q} \frac{p^{2}}{k} E_{k}^{M} H_{q}^{M}-j_{k p q} \frac{k p}{q} E_{q}^{M} H_{k}^{M} .
\end{aligned}
$$

The geometric coefficients $h_{k p q}, e_{k p q}$, and $j_{k p q}$ follow from the solenoidality constraints in Eq. (3) and are given in Ref. [10]. The triangle restricts integration to wave vectors $\mathbf{k}, \mathbf{p}$, and $\mathbf{q}$, which form a triangle, i.e., to a domain in the $p-q$ plane that is defined by $q=|\mathbf{p}+\mathbf{k}|$. The time $\theta_{k p q}$ is characteristic of the eddy damping of the nonlinear energy flux involving wave numbers $k, p$, and $q$. It is defined phenomenologically, but its particular form does not play a role in the following arguments.

The three nonlinear contributions on the right-hand side of Eq. (4) can be associated with the advective $T_{k}^{\mathrm{adv}}$ and explicitly twisting $T_{k}^{k h l}$ effects of turbulent fluctuations, as well as self-interaction ( $T_{k}^{\text {Lor }}$ ) of the magnetic field through the Lorentz force. Assuming that the most important nonlinearities involve the turbulent velocity and that the spectral scaling range of $H_{k}^{M}$ is stationary, a dynamical equilibrium of turbulent advection and the $H^{M}$-increasing effect of helical fluctuations is assumed, i.e. $T_{k}^{\mathrm{adv}} \sim T_{k}^{k h l}$. A dimensional approximation of the respective flux terms $k E_{k}^{K} H_{k}^{M} \sim k^{-1} E_{k}^{M} H_{k}^{K}$ yields

$$
H_{k}^{K} \sim\left(\frac{E_{k}^{K}}{E_{k}^{M}}\right)^{\gamma} k^{2} H_{k}^{M}, \quad \gamma=1
$$

This is a statement about the spectral dynamics of kinetic and magnetic helicity [or current helicity $H^{J}=\frac{1}{2 V} \int_{V}(\nabla \times \mathbf{b}) \cdot \mathbf{b}$ since $\left.H_{k}^{J} \sim k^{2} H_{k}^{M}\right]$ in the case of $E_{k}^{K} / E_{k}^{M} \neq 1$. The agreement of Eq. (5) with the numerical experiment is significantly improved [see Fig. 3(d)] when increasing $\gamma$ by one. This yields the main result

$$
H_{k}^{K} \sim\left(\frac{E_{k}^{K}}{E_{k}^{M}}\right)^{2} H_{k}^{J}
$$

Equation (6) is fulfilled, i.e., constant and close to unity, for almost all wave numbers $k>12$ excluding the drive and deep dissipation scales; however, it does not cover the full spectral scaling range of $H_{k}^{M}$ due to its susceptibility to the asymmetry of energies and helicities introduced by the large-scale energy sink. The higher-order modification of Eq. (5) cannot be motivated within the framework of quasinormal EDQNM theory using the chosen approach of nonlinear equilibrium. The underlying cause is presumably the nonlocality of the inverse-cascade process, which is not captured by the dimensional simplification of the EDQNM equations.

For verification purposes, a test simulation of decaying turbulence with the same numerical resolution of $1024^{3}$ and an initially finite level of magnetic helicity, i.e., $50 \%$ of the energetically possible maximum $H_{\max }^{M} \sim E^{M} / k_{0}$, is conducted. The initial Gaussian random-phase energy spectrum with $E_{k}^{K}=E_{k}^{M}$ for all $k$ is peaked around $k_{0}=70$ to allow some development of inverse transfer. The distribution of $H_{k}^{M}$ is homogeneous over the initial spectrum. The hyperdiffusive 


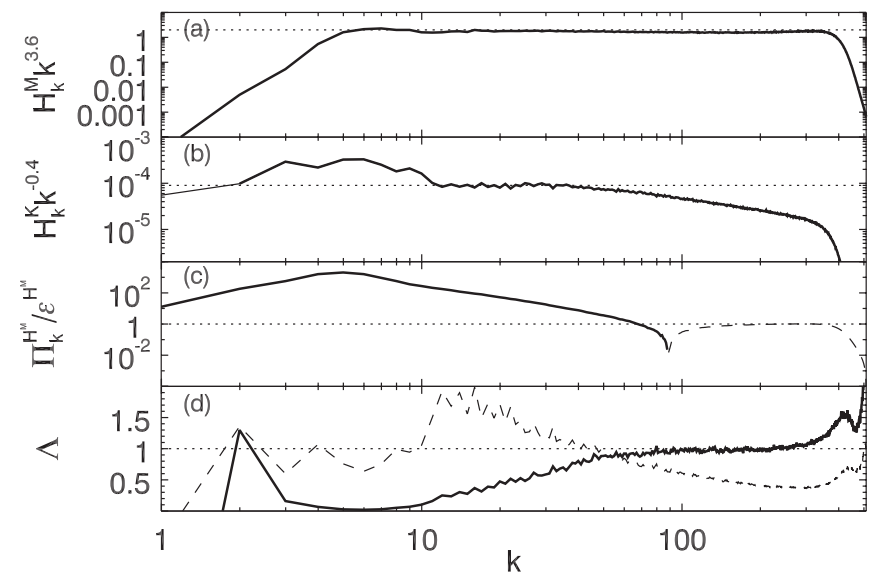

FIG. 4. Compensated spectra of (a) magnetic helicity, (b) kinetic helicity. (c) Spectral magnetic helicity flux normalized by corresponding dissipation rate (Inverse: solid curve, direct: dashed curve). (d) $\Lambda=\left(E_{k}^{K} / E_{k}^{M}\right)^{\gamma} H_{k}^{J} / H_{k}^{K}$ for $\gamma=1$ and scaled by a factor $1 / 3$ (dashed line) and for $\gamma=2$. All spectra shown have been taken at $t=9.2$ in the decaying simulation.

coefficients are chosen as $\mu_{8}=\eta_{8}=3 \times 10^{-41}$ with forcing switched off. Figure 4 displays the most important results of this decaying test simulation after about 9.2 large-eddy turnover times. The magnetic helicity [Fig. 4(a)] exhibits approximate self-similar spectral scaling $\sim k^{-3.6}$ with most of the excited scales in the range $15 \lesssim k \lesssim 60$ having developed during the decay. Kinetic helicity [Fig. 4(b)] reflects this as it is not injected into the system but generated via the Lorentz force during the inverse transfer of $H_{k}^{M}$. It shows two distinct asymptotic power-laws: $\sim k^{-0.5}$ for $60 \lesssim k \lesssim 120$ and $\sim k^{0.4}$ for $11 \lesssim k \lesssim 45$ in the range covered by the inverse transfer of $H_{k}^{M}$ during turbulence decay. The signature of kinetic, magnetic, and total energies is similar to the observation made in the driven case. The spectral flux of magnetic helicity [Fig. 4(c)] exhibits a split near $k_{0}$ as for $k>k_{0}$ small-scale dissipation determines the helicity transfer direction while for $k<k_{0}$ an inverse transfer with $k$-dependent $\Pi_{k}^{H^{M}}$ is observed. This is reflected by Eq. (6) [see Fig. 4(d)], which is fulfilled only in the region of approximately constant $\Pi_{k}^{H^{M}}$ and is apparently independent of the transfer direction.

A comparison of the presented findings with mean-field dynamo theory (see, for example, Ref. [3] and references therein), in particular the $\alpha$ coefficient including the current helicity $\alpha \sim H^{J}-H^{K}$, is interesting. Equation (6) is consistent with vanishing $\alpha$ since the cascade process does not generate magnetic flux and thus does not act as a dynamo itself. It furthermore suggests a modified $\alpha \sim\left(E^{K} / E^{M}\right)^{2} H^{J}-H^{K}$, which includes the squared ratio of kinetic and magnetic energies $\sim\left(E^{M}\right)^{-2}$ as a turbulent dynamo-quenching mechanism of the current helicity contribution to $\alpha$. This form of $\alpha$ quenching has recently been observed in a numerical test-field model [18].

In summary, a different and probably universal relation connecting the spectral behavior of magnetic and kinetic helicities and energies in homogeneous MHD turbulence is found motivated by statistical EDQNM closure results. Direct numerical simulations of MHD turbulence that is decaying or driven at small scales confirm the validity of the findings for spectral intervals of constant flux of magnetic helicity. The result has interesting connections to the $\alpha$ coefficient known from mean-field dynamo theory: It suggests an inherent and strong quenching of the dynamo, in particular of the current helicity effect, if the energy of turbulent magnetic fluctuations grows compared to the kinetic contribution of velocity.

The authors are grateful for discussions with A. Brandenburg, P. Diamond, U. Frisch, R. Grappin, P. Mininni, and A. Pouquet.
[1] Fluid Dynamics and Dynamos in Astrophysics and Geophysics, edited by A. M. Soward, C. A. Jones, D. W. Hughes, and N. O. Weiss, Durham Symposium on Astrophysical Fluid Mechanics 2002 (CRC, Boca Raton, FL, 2005).

[2] R. M. Kulsrud and E. G. Zweibel, Rep. Prog. Phys. 71, 046901 (2008).

[3] A. Brandenburg and K. Subramanian, Phys. Rep. 417, 1 (2005).

[4] H. K. Moffatt, J. Fluid Mech. 35, 117 (1969).

[5] M. A. Berger and G. B. Field, J. Fluid Mech. 147, 133 (1984).

[6] D. Biskamp, Nonlinear Magnetohydrodynamics (Cambridge University Press, Cambridge, 1993).

[7] L. Woltjer, Proc. Natl. Acad. Sci. USA 44, 833 (1958).

[8] U. Frisch, A. Pouquet, J. Léorat, and A. Mazure, J. Fluid Mech. 68, 769 (1975).
[9] A. Brandenburg, Space Sci. Rev. 144, 87 (2009).

[10] A. Pouquet, U. Frisch, and J. Léorat, J. Fluid Mech. 77, 321 (1976).

[11] A. Brandenburg, Astrophys. J. 550, 824 (2001).

[12] A. Alexakis, P. Mininni, and A. Pouquet, Astrophys. J. 640, 335 (2006).

[13] P. D. Mininni and A. Pouquet, Phys. Rev. E 80, 025401 (2009).

[14] W.-C. Müller and R. Grappin, Phys. Rev. Lett. 95, 114502 (2005).

[15] D. Biskamp and U. Bremer, Phys. Rev. Lett. 72, 3819 (1994).

[16] H. Aluie and G. L. Eyink, Phys. Rev. Lett. 104, 081101 (2010).

[17] W.-C. Müller and D. Biskamp, Phys. Rev. Lett. 84, 475 (2000).

[18] M. Rheinhardt and A. Brandenburg, Astron. Astrophys. 520, A28 (2010). 